\title{
Matérián
}

ISSN 1517-7076

Revista Matéria, v. 14, n. 2, pp. 869 - 877, 2009

http://www.materia.coppe.ufrj.br/sarra/artigos/artigo11080

\section{Avaliação da tenacidade à fratura através do ensaio de indentação em pastilhas de metal duro}

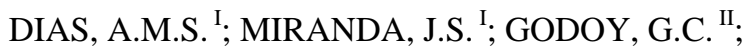 \\ I Departamento de Engenharia Mecânica - Universidade Federal de São João Del-Rei (UFSJ) \\ Praça Frei Orlando, 170 - CEP: 36.307-352, São João Del-Rei, MG. \\ e-mail: avelino@ufsj.edu.br, redajbv@yahoo.com.br \\ ${ }^{\mathrm{II}}$ Departamento de Engenharia Metalúrgica - UFMG - CEP: 30.160-030, Belo Horizonte, MG. \\ e-mail: gcgodoy@uaivip.com.br
}

\begin{abstract}
RESUMO
O teste de indentação Vickers tem sido muito utilizado para avaliação da dureza superficial em diferentes tipos de materiais. Devido à sua versatilidade, esta metodologia também é utilizada para avaliar diferentes propriedades mecânicas, como o módulo de Young $(E)$ e a tenacidade à fratura $\left(K_{I C}\right)$, principalmente em materiais sinterizados como, por exemplo, o carboneto de tungstênio com cobalto (WCCo). Este material é usado na fabricação de ferramentas de corte, uma vez que possui altas dureza e resistência à compressão, porém baixa tenacidade à fratura, diminuindo sua vida útil quando submetido a severas condições de desgastes. Entretanto, devido a essas características mecânicas peculiares, o WC-Co apresenta análise difícil através dos ensaios convencionais, principalmente para avaliação da tenacidade à fratura. Por isso, o objetivo do presente trabalho foi avaliar experimentalmente a dureza e a tenacidade à fratura de duas pastilhas comerciais de carboneto de tungstênio contendo cobalto a partir do ensaio Vickers. Um procedimento simples e barato de preparação da amostra consistiu de um polimento seletivo foi utilizado com o intuito de se evitar tensões residuais superficiais. Em seguida, avaliou-se a distribuição dos carbonetos de tungstênio na matriz de cobalto através da microscopia eletrônica de varredura (MEV). Os resultados desta análise mostraram uniformidade na dispersão do carboneto de tungstênio na matriz de cobalto, indicando que o processo de preparação da amostra não acarretou grandes tensões residuais superficiais. Determinaram-se os valores de dureza e de tenacidade à fratura das pastilhas estudadas e estes resultados se mostraram de acordo com os dados encontrados na literatura.
\end{abstract}

Palavras chaves: indentação Vickers, tenacidade à fratura, MEV.

\section{Evaluation of fracture toughness by indentation testing in hardmetal tools}

\section{ABSTRACT}

The Vickers indentation testing is commonly used for hardness surface measurement for different types of materials. This testing method is quite versatile and it is used to evaluate different mechanical properties, such as Young modulus (E) and fracture toughness (KIC), particularly in sintered materials like the tungsten carbide with cobalt (WC-Co). This material is used in the manufacturing of cutting tools, once it has very high surface hardness and also compression strength, although low fracture toughness values, which decreases its service life when submitted to high wear conditions. However, due to those peculiar mechanical properties, the WC-Co presents difficult analysis through conventional testing techniques, mainly considering fracture toughness evaluation. For that reason, the aim of this work was to evaluate experimentally the hardness and fracture toughness in two commercial tools, made of tungsten carbide with cobalt, through Vickers indentation testing. A simple and low cost procedure of sample preparation was used, consisted of a selective polishing, in order to avoid superficial residual stresses. In sequence, the scanning electron microscope (SEM) was used to evaluate the distribution of tungsten carbide in the cobalt matrix. The results of this analysis have shown uniformity in the tungsten carbide dispersion in the cobalt matrix, indicating that the sample preparation process did not create great values of superficial residual stresses. The experimental hardness and fracture toughness values were obtained, which have agreed with the ones found in the literature.

Keywords: Vickers indentation, fracture toughness, SEM. 


\section{INTRODUÇÃO}

Devido à sua versatilidade, o ensaio de indentação com penetradores piramidais tem sido estudado para avaliar propriedades mecânicas, como dureza superficial $(H)$, módulo de Young $(E)$, tenacidade à fratura $\left(K_{I C}\right)$ e uma curva de tensão em função da deformação para diferentes tipos de materiais [1]. Entretanto, a implementação desta técnica de indentação para a avaliação das propriedades mecânicas e os seus resultados obtidos ainda ocasionam dúvidas no meio científico. Estas dúvidas ainda são intensas quando se pretende avaliar as propriedades de materiais com características mecânicas peculiares como, por exemplo, o carboneto de tungstênio com cobalto (WC-Co). O WC-Co, que apesar de ser considerado frágil, apresenta um comportamento ligeiramente elasto-plástico sob compressão. O mesmo tem sido muito utilizado na confecção de ferramentas de corte para diferentes processos de fabricação. Nesse setor de fabricação mecânica, desejam-se materiais que apresentem alta dureza superficial aliada a grandes resistências à compressão e aos mecanismos de desgaste [2].

O desenvolvimento de materiais sinterizados, particularmente o carboneto de tungstênio com cobalto ocorreu em dois estágios. O primeiro, entre os anos entre 1920 e 1970, quando se focalizou o ganho de resistência mecânica através do aumento da dureza pela adição dos chamados multicarbonetos (TiC, TaC e $\mathrm{NbC}$ ), os quais também lhe conferiram estabilidade química [3]]. Em contrapartida ao ganho de dureza, ocorreu a perda de tenacidade de tais materiais, limitando a sua aplicação. Em um segundo estágio, a partir da década de 70, a atenção para o desenvolvimento do WC-Co foi direcionada para sua micro e nanoestrutura $[\underline{3}, \underline{4}$ ]. Estes autores propuseram o refinamento dos carbonetos, particularmente do WC, em forma (tamanho e geometria) bem como na sua dispersão na matriz de Co, buscando-se assim, homogeneidade de propriedades mecânicas e térmicas, típica de materiais considerados isotrópicos [픈. $\underline{4}$. Desta forma, o estudo destes materiais constitui objeto de investigações até os dias atuais.

Entretanto, o WC-Co se mostra difícil de ser analisado através de ensaios mecânicos convencionais, principalmente para avaliação de sua tenacidade à fratura. Por isso, nas duas últimas décadas alguns trabalhos foram desenvolvidos para avaliar a tenacidade do WC-Co a partir dos ensaios de indentação Vickers [ $\underline{5}-\underline{9}$ ]. Nestes trabalhos, os autores são unânimes em citar duas grandes dificuldades para obtenção de valores confiáveis para a tenacidade à fratura a partir deste ensaio. A primeira, devido à diversidade de equações experimentais encontradas na literatura, que utilizam dois possíveis modelos para os mecanismos de nucleação e propagação de trincas [10]. A outra dificuldade, devido ao surgimento do campo de tensões residuais superficiais oriundas da preparação da amostra [6]. A Figura 1 ilustra trincas radiais superficiais que surgiram durante o ensaio de dureza Vickers em um carboneto de tungstênio contendo 6\% de cobalto [11].

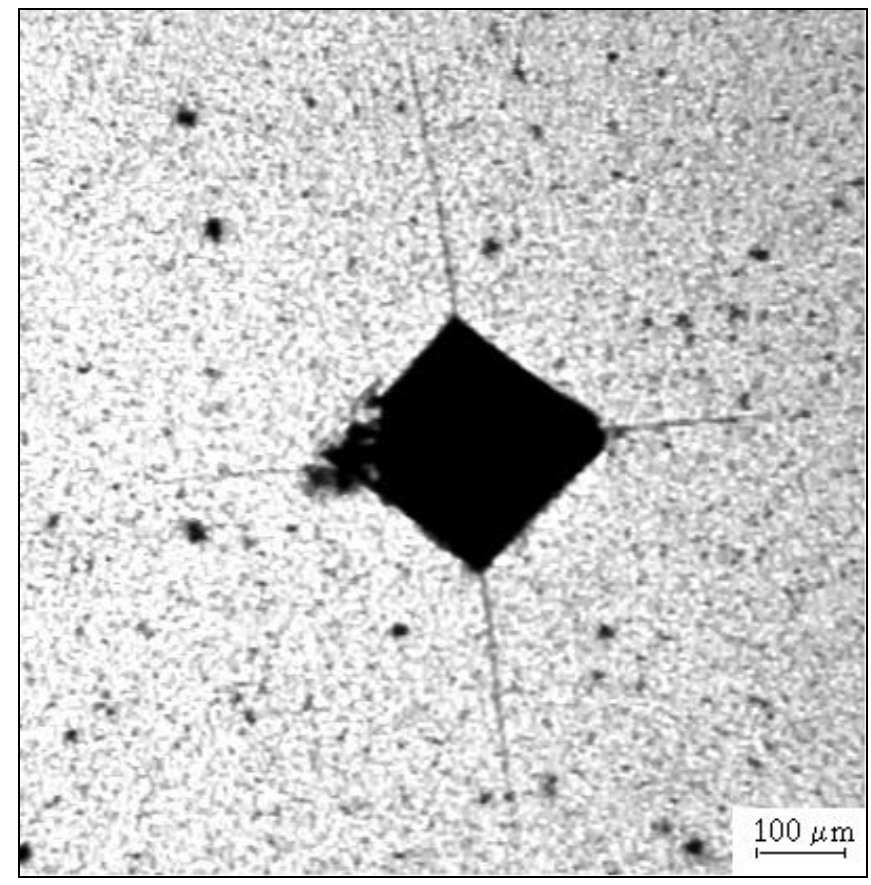

Figura 1: Trincas radiais superficiais em um WC-6Co [11]. 
O objetivo do presente trabalho foi estudar as propriedades de dureza e de tenacidade à fratura através do ensaio de indentação Vickers em duas pastilhas de ferramenta de corte de carboneto de tungstênio contendo de cobalto. Para evitar o surgimento de tensões residuais na preparação da amostra, adotou-se um procedimento metalográfico denominado polimento seletivo [12]. Utilizou-se a análise através da microscopia eletrônica de varredura (MEV) para avaliar a distribuição dos carbonetos de tungstênio na matriz de cobalto. Também se avaliou a composição química destas duas ferramentas de corte através da espectroscopia de energia dispersiva (EDS).

\section{MATERIAIS E MÉTODOS}

Foram realizados ensaios em amostras de ferramentas de corte feitas de carbonetos de tungstênio com cobalto. Estas amostras provêm das pastilhas CNMG 120408-15 GC 415 e RCMX 120400 P 35, fabricadas pela Sandvik ${ }^{\odot}$ e fornecidas pela Magneti-Marelli/COFAP ${ }^{\odot}$ para realização deste trabalho. A seguir, estão descritos os procedimentos usados na preparação da amostra, seguido da avaliação da uniformidade superficial das pastilhas e, finalmente, a descrição do ensaio de dureza e a avaliação da tenacidade à fratura nestas pastilhas.

As pastilhas supracitadas são recobertas pelo fabricante com um filme de nitreto de titânio (TiN) para melhorar suas propriedades tribológicas. Por isso, houve a necessidade de cortá-las ao meio para em seguida embuti-las em corpos rígidos de baquelite, possibilitando a análise do substrato de WC-Co. Foi possível obter quatro amostras da seção transversal de cada pastilha. Fez-se o lixamento da superfície das amostras com lixas impregnadas com carboneto de silício (SiC). Utilizaram-se lixas com as seguintes granulometrias 120, 240, 300, 400, 500, 600, 800, 1000, 1200, 1400 e 1500. Iniciou-se o procedimento de lixamento a partir da lixa de maior granulomentria (120) até a de menor, sucessivamente. O tempo total de lixamento foi de aproximadamente uma hora. Posteriormente, através de um polimento com alumina (Al2O3) em suspensão aquosa na menor granulometria disponível $(0,03 \mu \mathrm{m})$, prepararam-se as amostras para serem ensaiadas. Neste polimento seletivo, aplicou-se manualmente uma leve carga com um movimento alternado de rotação em torno do eixo da amostra [12]. Não houve necessidade da utilização de reagentes específicos durante o polimento. Esta técnica metalográfica foi elaborada com o objetivo de acarretar o menor nível de tensões residuais na superfície da amostra, pois os procedimentos convencionais não se mostraram eficientes para este propósito. Fato este que pode ser atribuído à severidade da técnica convencional. Outras vantagens deste procedimento metalográfico foram sua simplicidade e seu baixo custo, não sendo necessário nem a prévia metalização das amostras para posterior visualização no microscópio eletrônico de varredura (MEV).

Realizaram-se os ensaios experimentais de indentação utilizando uma máquina de ensaio de dureza HECKERT $^{\circledR}$ e um penetrador de diamante piramidal Vickers de base quadrada, com ângulo de abertura entre as faces de $136^{\circ}$. A carga utilizada nestes foi de 588,6 N (60 Kgf), segundo as orientações da norma DIN 50133 [13]. Esta carga foi lentamente aplicada na superfície da amostra por meio de um pistão e mantida durante 30 segundos, sendo depois retirada e sua impressão observada através de um microscópio ótico [14]. O objetivo desta análise experimental foi determinar a dureza superficial da amostra e avaliar sua tenacidade à fratura a partir de equações semi-empíricas encontradas na literatura especializada [10, 15]. Figura 2 mostra um esquema da impressão do ensaio Vickers, assim como, ilustra a dimensão da diagonal do indentador (2a) e o comprimento das trincas radiais $(l)$.

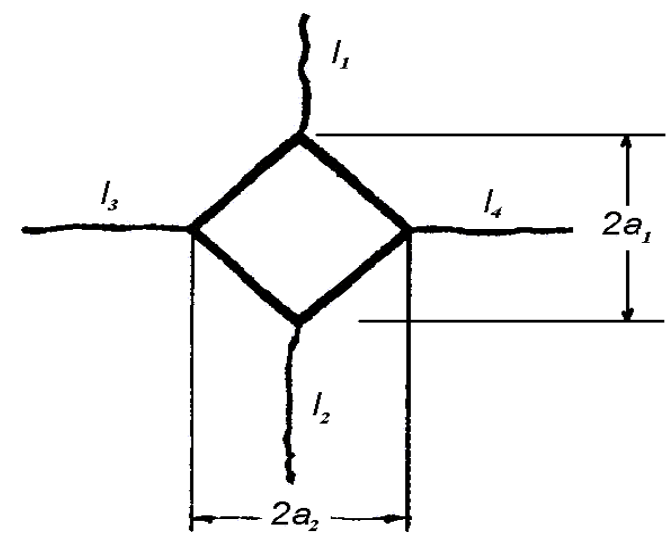

Figura 2: Representação esquemática das medidas das trincas radiais Palmqvist $[11,15]$. 
Para avaliar a tenacidade à fratura a partir do ensaio Vickers, utilizou-se a expressão semi-empírica para trincas radiais Palmqvist descrita na Equação (1) [6, 7, 10, 15].

$$
\left(\frac{K_{I C} \phi}{H a^{1 / 2}}\right)\left(\frac{H}{E \phi}\right)^{2 / 5}=0,035\left(\frac{l}{a}\right)^{-1 / 2}
$$

Nesta, KIC é a tenacidade à fratura do material, H é a dureza superficial da amostra, E é o seu módulo de Young, a é a metade da impressão da diagonal do indentador e l é o comprimento da trinca radial, conforme ilustrado na Figura 2. Segundo a literatura, se a relação entre o comprimento médio das trincas radias (l), dividida pela metade da diagonal da impressão do indentador (a), estiver dentro da faixa de $0,25 \leq 1 / a \leq 2,50$, estas trincas radiais superficiais apresentam-se do tipo Palmqvist [6, 7, 10 e 15]. Esta Equação (1) pode ser reescrita, Equação (2), considerando-se o fator de restrição, $\phi$ igual a 2,70 [10].

$$
K_{I C}=0,019(\mathrm{Ha})\left(\frac{E}{H}\right)^{2 / 5} l^{-1 / 2}
$$

O sistema de trinca Palmqvist tem sido verificado nos ensaios Vickers em amostras de WC com diferentes percentuais de Co $[\underline{6}, \underline{11}, \underline{15}]$. Szutkowska (1999) estudou os WC-Co, pois, segundo ele, existe um grande interesse da indústria de fabricação mecânica na utilização de novas metodologias confiáveis para avaliação da tenacidade à fratura nestes carbonetos [6] . Este autor também chamou a atenção para a influência negativa das tensões residuais provenientes da preparação da amostra sobre os resultados de tenacidade obtidos através das Equações (1) e (2).

\section{RESULTADOS E DISCUSSÕES}

Após a preparação das amostras através da técnica do polimento seletivo, avaliaram-se a morfologia e o aspecto microestrutural dos carbonetos dispersos na matriz de cobalto. As Figuras 3 e 4 apresentam, respectivamente, as fotomicrografias das amostras das pastilhas GC 415 e P 35.

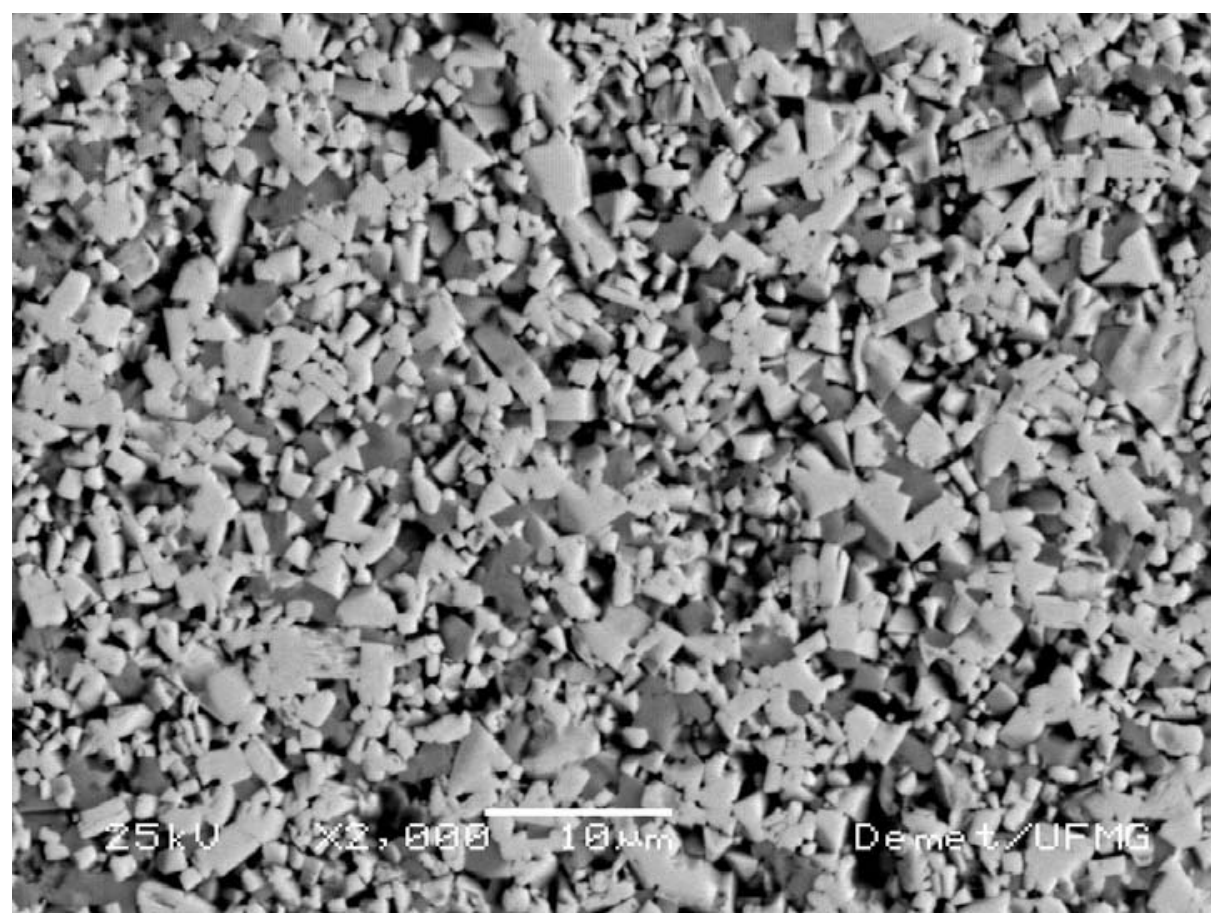

Figura 3: Microestrutura da amostra da pastilha CNMG 120408-15 GC 415.

Analisando-se estas figuras, verifica-se que estas duas pastilhas apresentam uniformidade em relação à distribuição dos carbonetos na matriz de cobalto. O fabricante, inclusive, atesta que estas ferramentas são equilibradas no âmbito de suas propriedades mecânicas devido à distribuição e à proporção adequadas de seus constituintes (carbonetos e cobalto) [16]. Estas fotomicrografias também mostram que a técnica do polimento seletivo não arrancou os carbonetos de tungstênio da superfície da amostra, devido à 
sua baixa severidade, não produzindo tensões residuais superficiais significativas durante este processo de preparação da amostra. Também se observa que a pastilha P 35, Figura 4, apresentou microestrutura dos carbonetos mais refinada, se comparada à pastilha GC 415, Figura 3.

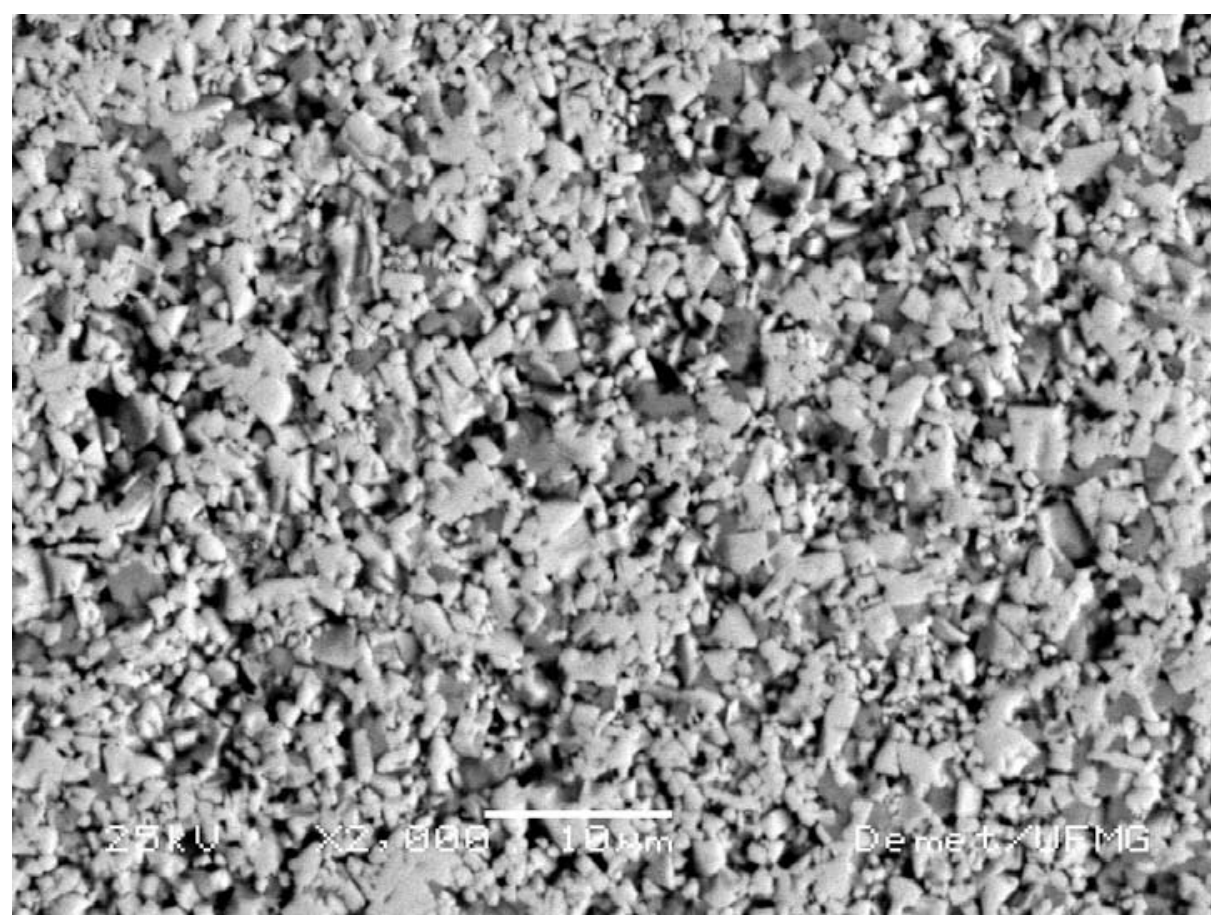

Figura 4: Microestrutura da amostra da pastilha RCMX 120400 P 35.

A Tabela 1 ilustra os percentuais volumétricos de carboneto de tungstênio e de cobalto nas amostras das ferramentas GC 415 e P 35, respectivamente, obtidos através da espectroscopia de energia dispersiva. Com base nestes resultados, estima-se que a pastilha GC 415 seja confeccionada com $6 \%$ de cobalto (WC6Co) e a P 35 contém 12,5 \% de cobalto (WC-12,5Co). Conforme a literatura, o aumento do percentual de cobalto no metal duro, diminui sua dureza aumentando sua tenacidade à fratura [5].

Tabela 1: Determinação dos percentuais de WC e de Co nas pastilhas estudadas através de EDS.

\begin{tabular}{|c|c|c|c|}
\hline \multirow{2}{*}{ Pastilha } & Amostra & \% vol. WC & \% vol. Co \\
\hline \multirow{4}{*}{ GC 415 } & 1 & 95,15 & 4,85 \\
\cline { 2 - 4 } & 2 & 93,87 & 6,13 \\
\cline { 2 - 4 } & 3 & 91,64 & 8,36 \\
\cline { 2 - 4 } & 4 & 92,59 & 7,41 \\
\hline \multirow{5}{*}{ P 35 } & 1 & 85,05 & 14,95 \\
\cline { 2 - 4 } & 2 & 95,89 & 11,79 \\
\cline { 2 - 4 } & 3 & 86,86 & 13,14 \\
\cline { 2 - 4 } & 4 & 88,10 & 11,90 \\
\hline
\end{tabular}

A Tabela 2 apresenta os dados experimentais obtidos nos ensaios de dureza realizados nas amostras das duas pastilhas. Devido à pequena dimensão das mesmas e, conseqüentemente, das quatro amostras de cada pastilha, realizaram-se apenas dois ensaios em cada uma das amostras. A dureza superficial média encontrada foi de 15,70 \pm 0,63 GPa para a pastilha GC 415 e de 15,09 \pm 0,12 GPa para a P 35. Foi verificado que estes valores experimentais encontram-se dentro dos limites disponíveis na literatura para carbonetos de tungstênio com 5,00 - 15,00\% de cobalto, e da faixa descrita pelo fabricante (14,00 a 18,00 GPa) [모 $\underline{10}, \underline{16}]$. Observou-se uma uniformidade da dureza superficial para cada pastilha. Observou-se também uma proporcionalidade entre a diagonal do indentador $(a)$ e a tamanho da trinca radial (l), Figura 2. Estas 
observações confirmam o equilíbrio no âmbito das propriedades mecânicas destas pastilhas, conforme informado pelo fabricante [16].

Comparando-se os valores de dureza destas pastilhas, constatou-se que estes resultados foram muitos semelhantes, embora as pastilhas apresentarem diferentes percentuais do elemento ligante (cobalto). Entretanto, a pastilha P 35 apresentou uma microestrutura de carbonetos de tungstênio mais refinada, Figura 4, acarretando em um aumento na dureza. Em contrapartida, esta mesma pastilha tem um maior percentual de cobalto, Tabela 1, fato que diminui sua dureza. Em resumo, variando-se o tamanho de grão, concomitantemente ao aumento do percentual de cobalto, o fabricante produziu duas diferentes pastilhas com dureza similares.

Tabela 2: Dados experimentais obtidos no ensaio de indentação Vickers.

\begin{tabular}{|c|c|c|c|c|c|}
\hline Pastilha & Amostra & $\begin{array}{c}\text { Comprimento } \\
\text { médio da diagonal }\end{array}$ & $\begin{array}{c}\text { Comprimento médio } \\
\text { trinca radial }\end{array}$ & $1 / a$ & Dureza $(H)$ \\
\hline \multirow{8}{*}{ GC 415} & \multirow{2}{*}{1} & $\mathrm{a}_{1}=134,00 \mu \mathrm{m}$ & $l_{1-2}=290,50 \mu \mathrm{m}$ & 2,17 & \multirow{2}{*}{$15,20 \mathrm{GPa}$} \\
\hline & & $\mathrm{a}_{2}=134,00 \mu \mathrm{m}$ & $l_{3-4}=250,30 \mu \mathrm{m}$ & 1,87 & \\
\hline & \multirow{2}{*}{2} & $\mathrm{a}_{1}=131,00 \mu \mathrm{m}$ & $l_{1-2}=304,30 \mu \mathrm{m}$ & 2,32 & \multirow{2}{*}{$15,84 \mathrm{GPa}$} \\
\hline & & $\mathrm{a}_{2}=131,50 \mu \mathrm{m}$ & $l_{3-4}=275,80 \mu \mathrm{m}$ & 2,10 & \\
\hline & \multirow{2}{*}{3} & $\mathrm{a}_{1}=132,00 \mu \mathrm{m}$ & $l_{1-2}=293,10 \mu \mathrm{m}$ & 2,22 & \multirow{2}{*}{$15,66 \mathrm{GPa}$} \\
\hline & & $\mathrm{a}_{2}=132,00 \mu \mathrm{m}$ & $l_{3-4}=290,20 \mu \mathrm{m}$ & 2,20 & \\
\hline & \multirow{2}{*}{4} & $a_{1}=129,00 \mu \mathrm{m}$ & $l_{1-2}=268,70 \mu \mathrm{m}$ & 2,08 & \multirow{2}{*}{$16,09 \mathrm{GPa}$} \\
\hline & & $\mathrm{a}_{2}=131,50 \mu \mathrm{m}$ & $l_{3-4}=250,60 \mu \mathrm{m}$ & 1,91 & \\
\hline \multirow{8}{*}{ P 35} & \multirow{2}{*}{1} & $\mathrm{a}_{1}=134,00 \mu \mathrm{m}$ & $l_{1-2}=270,20 \mu \mathrm{m}$ & 2,02 & \multirow{2}{*}{$15,08 \mathrm{GPa}$} \\
\hline & & $\mathrm{a}_{2}=135,00 \mu \mathrm{m}$ & $l_{3-4}=293,40 \mu \mathrm{m}$ & 2,17 & \\
\hline & \multirow{2}{*}{2} & $\mathrm{a}_{1}=136,00 \mu \mathrm{m}$ & $l_{1-2}=286,65 \mu \mathrm{m}$ & 2,11 & \multirow{2}{*}{$14,81 \mathrm{GPa}$} \\
\hline & & $\mathrm{a}_{2}=135,50 \mu \mathrm{m}$ & $l_{3-4}=252,00 \mu \mathrm{m}$ & 1,86 & \\
\hline & \multirow{2}{*}{3} & $\mathrm{a}_{1}=132,30 \mu \mathrm{m}$ & $l_{1-2}=270,60 \mu \mathrm{m}$ & 2,05 & \multirow{2}{*}{$15,22 \mathrm{GPa}$} \\
\hline & & $\mathrm{a}_{2}=135,50 \mu \mathrm{m}$ & $l_{3-4}=275,90 \mu \mathrm{m}$ & 2,04 & \\
\hline & \multirow{2}{*}{4} & $\mathrm{a}_{1}=133,50 \mu \mathrm{m}$ & $l_{1-2}=270,15 \mu \mathrm{m}$ & 2,02 & \multirow{2}{*}{$15,25 \mathrm{GPa}$} \\
\hline & & $\mathrm{a}_{2}=134,00 \mu \mathrm{m}$ & $l_{3-4}=277,25 \mu \mathrm{m}$ & 2,07 & \\
\hline
\end{tabular}

Na Tabela 2 também foi calculada a relação entre o comprimento médio das trincas radias dividida pela metade da diagonal da impressão do indentador. Se esta relação $(l / a)$ estiver dentro do intervalo entre $0,25$ e 2,50, as fissuras superficiais são classificadas de trincas radiais Palmqvist [6, $\underline{7}, \underline{10}, \underline{15}]$. Assim sendo, constatou-se que todas as amostras das duas pastilhas apresentaram trincas radias Palmqvist na direção da diagonal da impressão do indentador. A partir dos resultados mostrados na Tabela 2, também se avaliou o valor da tenacidade à fratura $\left(K_{I C}\right)$ através da Equação (2). Nesta, adotou-se o valor do módulo de Young do WC-6Co ( $E=619,5 \mathrm{GPa})$ encontrado por Zeng e Chiu, através da técnica da indentação instrumentada [17].

A Tabela 3 apresenta os valores calculados da tenacidade à fratura para as amostras das duas pastilhas de ferramenta de corte. Os valores médios da tenacidade à fratura para as pastilhas GC 415 e P 35 foram, respectivamente, $10,28 \pm 0,40 \mathrm{MPa} * \mathrm{~m}^{1 / 2}$ e $10,29 \pm 0,22 \mathrm{MPa} * \mathrm{~m}^{1 / 2}$. Verifica-se que estes valores encontram-se dentro da faixa encontrada na literatura para pastilhas de carbonetos de tungstênio com 5 - 15\% de cobalto $[\underline{5}, \underline{10}, \underline{16}]$. Para fins de comparação, a tenacidade à fratura encontrada na literatura para o WC6Co foi de $10 \mathrm{MPA} * \mathrm{~m}^{1 / 2}$, a partir do ensaio de flexão e três pontos (SENB) [2]. O resultado encontrado para a pastilha GC 415 (WC-6Co) apresentou uma diferença de em torno de 3,8\% quando comparado com o valor de $K_{I C}$ da literatura.

Constatou-se também que estes resultados de tenacidade foram muitos semelhantes para as duas pastilhas que apresentam diferentes percentuais de cobalto. Porém, conforme anteriormente citado, ao variar o tamanho de grão concomitantemente com aumento do percentual de cobalto, o fabricante produziu duas diferentes pastilhas com propriedades mecânicas próximas. Isto mostra que os valores encontrados para 
dureza e para $K_{I C}$ destas ferramentas a partir dos ensaios de indentação foram adequados. Segunda a literatura, também se verificou que estas pastilhas apresentaram alta tenacidade à fratura []ㅡ.

Na literatura também foi possível encontrar outras metodologias para avaliação da tenacidade à fratura em materiais frágeis em detrimento do ensaio de indentação como, por exemplo, o ensaio de flexão em corpo de prova com entalhe V (SEVNB) [18]. Contudo, a confecção de amostras para o ensaio SEVNB não pôde ser realizada no presente trabalho, devido ao reduzido tamanho das pastilhas de ferramenta de corte.

Tabela 3: Avaliação da tenacidade a partir do ensaio Vickers.

\begin{tabular}{|c|c|c|c|c|c|}
\hline Pastilha & Dureza & $(E / H)^{2 / 5}$ & $a \mu \mathrm{m}$ & $l \mu \mathrm{m}$ & $K_{I C} \mathrm{MPa} \sqrt{\mathrm{m}}$ \\
\hline \multirow{8}{*}{ GC 415} & \multirow{2}{*}{$15,20 \mathrm{GPa}$} & \multirow{2}{*}{4,406} & 134,00 & 290,50 & 10,01 \\
\hline & & & 134,00 & 250,30 & 10,78 \\
\hline & \multirow{2}{*}{$15,84 \mathrm{GPa}$} & \multirow{2}{*}{4,334} & 131,00 & 304,30 & 9,80 \\
\hline & & & 131,50 & 275,80 & 10,33 \\
\hline & \multirow{2}{*}{$15,66 \mathrm{GPa}$} & \multirow{2}{*}{4,354} & 132,00 & 293,10 & 9,99 \\
\hline & & & 132,00 & 290,20 & 10,04 \\
\hline & \multirow{2}{*}{$16,09 \mathrm{GPa}$} & \multirow{2}{*}{4,307} & 129,00 & 268,70 & 10,36 \\
\hline & & & 131,50 & 250,60 & 10,94 \\
\hline \multirow{8}{*}{ P 35} & \multirow{2}{*}{$15,08 \mathrm{GPa}$} & \multirow{2}{*}{4,420} & 134,00 & 270,20 & 10,33 \\
\hline & & & 135,00 & 293,40 & 9,98 \\
\hline & \multirow{2}{*}{$14,81 \mathrm{GPa}$} & \multirow{2}{*}{4,452} & 136,00 & 286,65 & 10,06 \\
\hline & & & 135,50 & 252,00 & 10,69 \\
\hline & \multirow{2}{*}{$15,22 \mathrm{GPa}$} & \multirow{2}{*}{4,404} & 132,30 & 270,60 & 10,24 \\
\hline & & & 135,50 & 275,90 & 10,39 \\
\hline & \multirow{2}{*}{$15,25 \mathrm{GPa}$} & \multirow{2}{*}{4,401} & 133,50 & 270,15 & 10,36 \\
\hline & & & 134,00 & 277,25 & 10,26 \\
\hline
\end{tabular}

\section{CONCLUSÕES}

Neste trabalho foram apresentados os resultados experimentais obtidos no estudo de duas pastilhas de ferramentas de corte feitas de carboneto de tungstênio com cobalto. O procedimento metalográfico, denominado de polimento seletivo, elaborado com o propósito de não produzir tensões residuais superficiais significativas durante este processo de preparação da amostra, mostrou-se eficiente. Este polimento com óxido de alumínio na menor granulometria disponível $(0,03 \mu \mathrm{m})$ não arrancou os carbonetos de tungstênio das amostras e, através de uma análise por microscopia eletrônica de varredura, avaliou-se a homogeneidade da distribuição dos carbonetos de tungstênio dispersos na matriz de cobalto.

Desta forma, avaliou-se a tenacidade à fratura das amostras a partir dos resultados obtidos pelo ensaio de dureza Vickers através de uma expressão semi-empírica, sem a necessidade da utilização de um processo de recozimento para aliviar as tensões residuais superficiais na amostra. Os valores da dureza superficial e da tenacidade à fratura encontraram-se dentro da faixa de valores obtidos na literatura.

No presente trabalho também foi possível verificar as características dos carbonetos de tungstênio contendo cobalto utilizados na confecção das pastilhas estudadas. Estes materiais analisados apresentaram altos valores de dureza e de tenacidade à fratura, assim como homogeneidade na distribuição dos carbonetos. Todavia, ainda é possível encontrar carbonetos de tungstênio com cobalto com valores de tenacidade à fratura superiores aos encontrados para as pastilhas GC 415 e P 35. Finalmente, pode-se dizer que polimento empregado na preparação das amostras apresentou simplicidade e baixo custo, permitindo aplicar a metodologia do ensaio Vickers para determinação da tenacidade à fratura em pastilhas de ferramenta de corte. 


\section{AGRADECIMENTOS}

Os autores agradecem ao CNPq e a FAPEMIG (PRONEX EDT531/05) pelo suporte financeiro. Agradecimentos também ao Técnico Francisco Marcelino da Silva pelo inestimável auxílio na realização dos ensaios experimentais, ao Departamento de Metalurgia da UFMG por disponibilizar o uso do MEV e do EDS e a Magneti-Marelli/COFAP ${ }^{\mathscr{C}}$ pela doação das pastilhas de ferramentas de corte.

\section{REFERÊNCIAS BIBLIOGRAFICAS}

[1] DIAS, A.M.S., MODENESI, P.J., CRISTINA, G.C., "Numerical study of indentation testing in superficial coatings", In: Proceedings of International Congress in Mechanical Engineering, Brasília, November 2007 [CD-ROM].

[2] TRENT, E. M., Metal Cutting, 2 ed., London, Butterworths \& Co. LTD,1984.

[3] BALDONI, J.G., "State of the art in coated carbides", The carbide and tool journal, v. 12, n. 5, pp. $26-$ 29, November-December 1980.

[4] LEYLAND, A., MATTHEWS, A., "On the significance of the $\mathrm{H} / \mathrm{E}$ ratio in wear control: a nanocomposite coating approach to optimized tribological behaviour”, Wear, v. 246, n. 1-2, pp. 1 11, November 2000.

[5] SCHUBERT, W. D., NEUMEISTER, H., KINGER, G., LUX, B., "Hardness to toughness relationship of fine-grain WC-Co hardmetals”, International Journal of Refractory Metals \& Hard Metals, v. 16, n. 2, pp. 133-142, 1998.

[6] SZUTKOWSKA, M., "Fracture toughness measurement of WC-Co hardmetals by indentation method", Journal of Advanced Materials, v. 31, n. 3, pp. 3-7, July 1999.

[7] DIAS, A.M.S., MODENESI, P.J., CRISTINA, G.C., "Computer simulation of stress distribution during vickers hardness testing of WC-Co”, Materials Research, v. 9, n. 1, pp. 74-76, January-March 2006.

[8] SHETTY, D. K., WRIGHT, I. G., MINER, P. N., CLAUER, A. H., "Indentation fracture of WC-Co cermets”, Journal of Materials Science, v. 20, n. 5, pp. 1873-1882, May 1985.

[9] DENSLEY, J.M., HIRTH J. P., "Fracture toughness of a nanoscale WC-Co Toll Steel", Scripta Materialia, v. 38, n. 2, pp. 239-244, 1998.

[10] PONTON, C.B., RAWLINGS, R.D., "Vickers indentation fracture toughness test, Part 1: Application and evaluation of standardized indentation toughness equations", Materials Science and Technology, v. 5, pp. 961-976, September 1989.

[11] DIAS, A.M.S., Análise numérica do processo de fratura no ensaio de indentação vickers em uma Liga de WC-Co, Tese de D.Sc, PGEMM/UFMG, Brasil, 2004.

[12] AVILA, R.F., Desempenho de ferramentas de metal duro revestidas com Ti-N, Ti-C-N e Ti-Al-N no torneamento do aço ABNT 4340 temperado e revenido, Tese de D.Sc, PGEMM/UFMG, Brasil, 2003.

[13] Testing of metallic materials - Vickers Hardness Testing, http://www.normug.din.de. Acessado em Agosto de 2009.

[14] SOUZA, S.A., Ensaios mecânicos de materiais metálicos: fundamentos teóricos e prático,. 5 ed., São Paulo, Edgard Blücher, 286p, 2000.

[15] NIIHARA, K., "A fracture mechanics cnalysis of indentation-induced palmqvist crack in ceramics", Journal of the Materials Science Letters, v. 2, n. 5, pp. 221-223, May 1983.

[16] SANDVIK ${ }^{\odot}$, Manual do Fabricante, 2000. 
[17] ZENG, K., CHIU, C-h., “An analysis of Load-Penetration curves from instrumented indentation”, Acta Materialia, v. 49, n. 17, pp. 3539-3551, October 2001.

[18] STRECKER, K., RIBEIRO, S., HOFFMAN, M. J., "Fracture toughness measurements of LPS_SiC: A comparison of the indentation technique and the SEVNB method", Materials Research, v. 8, pp. 121-124, 2005. 